\title{
Composição e abundância relativa dos mamíferos terrestres de médio e grande porte do Parque Estadual da Serra do Brigadeiro, Minas Gerais, Brasil
}

\author{
André Valle Nunes ${ }^{1 *}$ \\ Gisele Lessa $^{2}$ \\ Leandro Moraes Scoss ${ }^{3}$ \\ ${ }^{1}$ PPG em Biologia Animal, Universidade Federal de Viçosa, Viçosa - MG, Brasil \\ ${ }^{2}$ Museu de Zoologia João Moojen, Universidade Federal de Viçosa, Viçosa - MG, Brasil \\ ${ }^{3}$ Vale S.A., Belo Horizonte - MG, Brasil \\ * Autor para correspondência \\ biovalle@yahoo.com.br \\ Museu de Zoologia João Moojen, Vila Gianetti 32, \\ CEP 36570-000, Viçosa - MG, Brasil
}

Submetido em 02/02/2012

Aceito para publicação em 26/04/2012

\section{Resumo}

Três áreas no norte do Parque Estadual da Serra do Brigadeiro (PESB) foram amostradas em relação à fauna de mamíferos terrestres de médio e grande porte, através de armadilhas fotográficas, ao longo de 12 meses. Foram registradas nove espécies silvestres e uma doméstica, a partir de 46 registros. A composição da comunidade foi baseada numa série exponencial com dominância de poucas espécies. Em contraposição, houve uma proporção relativamente alta de espécies que seriam "raras" para a comunidade observada, com dominância da onça-parda (Puma concolor) sobre as demais espécies. Os resultados demonstram que a área denominada Vale Perdido é a mais importante para a conservação da mastofauna, pois nessa área a estimativa de riqueza de espécies é maior $(13,99 \pm 2,23)$ se comparada com a das outras duas áreas, que tiveram uma riqueza de 6,00 $\pm 1,41$ e 6,00 $\pm 1,00$, respectivamente. É possível que características estruturais e espaciais do Vale Perdido favoreçam o uso dessa área pelas espécies. A estrutura da comunidade de mamíferos terrestres de médio e grande porte do PESB encontra-se desestruturada ou em fase de estruturação. Possivelmente, esse modelo foi ocasionado por efeitos históricos negativos da ocupação e do extrativismo que a região sofreu na década 1960 .

Palavras-chave: Armadilhas fotográficas; Conservação; Fragmentação; Mastofauna; Mata Atlântica

\section{Abstract}

Composition and relative abundance of terrestrial medium- and large-sized mammals of Parque Estadual da Serra do Brigadeiro, Minas Gerais, Brazil. Three northern areas of Parque Estadual da Serra do Brigadeiro (PESB) were sampled for terrestrial medium- and large-sized mammals, using camera traps, for twelve months. Nine wild species and one domestic species were registered, through 49 records. The community's composition was based on an exponential series with dominance of few species. In contrast, there was a relatively high proportion of species that might be "rare" in the community observed, with dominance of puma (Puma concolor) over the other ones. The results show that the area named Vale Perdido is the most 
important one for the conservation of mammals, because the estimated species richness is higher there (13.99 \pm 2.23 ) when compared to that of the other two areas, which had a richness of $6.00 \pm 1.41$ and $6.00 \pm 1.00$, respectively. It's possible that the structural and spatial characteristics of Vale Perdido favored the use of this area by the species. The structure of the community of medium-and large-sized terrestrial mammals of PESB is unstructured or in a structuring phase. Possibly, this model was caused by negative historical effects of the occupation and extraction that the region underwent in the 1960s.

Key words: Atlantic rainforest; Camera traps; Conservation; Fragmentation; Mammals

\section{Introdução}

Comunidades biológicas são usualmente definidas como associações entre espécies e populações que coexistem no espaço e no tempo, onde a riqueza, composição de espécies, abundância absoluta e relativa, distribuição espacial e atributos ecológicos são os principais parâmetros utilizados para descrever suas estruturas (BEGON et al., 1990). Variações existentes na sua organização podem ser resultantes de diferenças nas condições ambientais e ecológicas (WEBB et al., 2002).

Entre as comunidades de vertebrados que são mais afetadas pelas alterações das condições ambientais e ecológicas podem se destacar as formadas por espécies de mamíferos de médio e grande porte (HENLE et al., 2004). Em sua maioria, as espécies desse grupo necessitam de áreas de vida relativamente grandes (HASKELL et al., 2002; KIE et al., 2002), além de se mostrarem frágeis às mudanças da matriz do entorno dos remanescentes florestais (LAURANCE; BIERREGAARD, 1997; LAURANCE, 1999) e naturalmente por apresentarem densidades populacionais baixas (NOSS et al., 1996; PARDINI et al., 2005). Além disso, este grupo sofre com a alta pressão de caça, já que essa atividade ainda ocorre na maioria dos biomas brasileiros (CHIARELLO, 2000; PERES, 2001) e com a competição direta de espécies domésticas (p. ex. com Canis familiaris - FROLICH et al., 2000; FIORELLO et al., 2006).

Diante disso, a desestruturação de comunidades de mamíferos de médio e grande porte é preocupante, pois a ausência ou a baixa abundância de espécies desse grupo em fragmentos florestais acarreta mudanças no padrão espacial de regeneração e composição da vegetação (DIRZO; MIRANDA, 1990; CUARÓN, 2000; GALETTI et al., 2003) em função do papel que estes desempenham na dispersão e predação de sementes e plântulas.

Dentro deste contexto, os objetivos deste trabalho foram: (1) identificar a riqueza de espécies e a abundância relativa para três áreas do P. E. da Serra do Brigadeiro, (2) discutir os efeitos que atuam sobre a estrutura da comunidade e composição de espécies de mamíferos de médio e grande porte e, (3) testar a hipótese de que existe diferença na riqueza de espécies entre os sítios de amostragem.

\section{Material e Métodos}

\section{Área de estudo}

Os dados foram coletados em áreas de Mata Atlântica na Zona da Mata Mineira, região norte do Parque Estadual da Serra do Brigadeiro (PESB) nos municípios de Serecita, Pedra Bonita, Divino e Fervedouro (Figura 1) (42 $40^{\prime}$ e $40^{\circ} 20^{\prime} \mathrm{W}$ e $20^{\circ} 33^{\prime}$ e $\left.21^{\circ} 00^{\prime} \mathrm{S}\right)$. O parque apresenta uma área equivalente a 14.984ha e está inserido no conjunto de serras do Maciço da Mantiqueira entre os vales dos rios Carangola, Glória e Doce. Seu relevo é bastante acidentado por escarpas e maciços com grandes áreas de rocha aflorada, e a altitude varia de 860 a $1.985 \mathrm{~m}$ acima do nível do mar (CAIFA; SILVA, 2005).

A vegetação do PESB é composta por fragmentos secundários de Floresta Estacional Semidecidual e Floresta Ombrófila Densa (RIBEIRO, 2003) pertencentes ao domínio da Mata Atlântica. Também são encontrados Campos de Altitude, que ocupam platôs e escarpas isoladas em afloramentos rochosos acima de $1.600 \mathrm{~m}$, apresentando bom estado de conservação, sendo $10 \%$ da área do parque composta por esse tipo de fitofisionomia 
FIGURA 1: Localização das áreas de estudo (1 - Vale Perdido, 2 - Matipó, 3 - Ararica) na porção norte do Parque Estadual da Serra do Brigadeiro, localizado na América do Sul, Minas Gerais, Brasil.

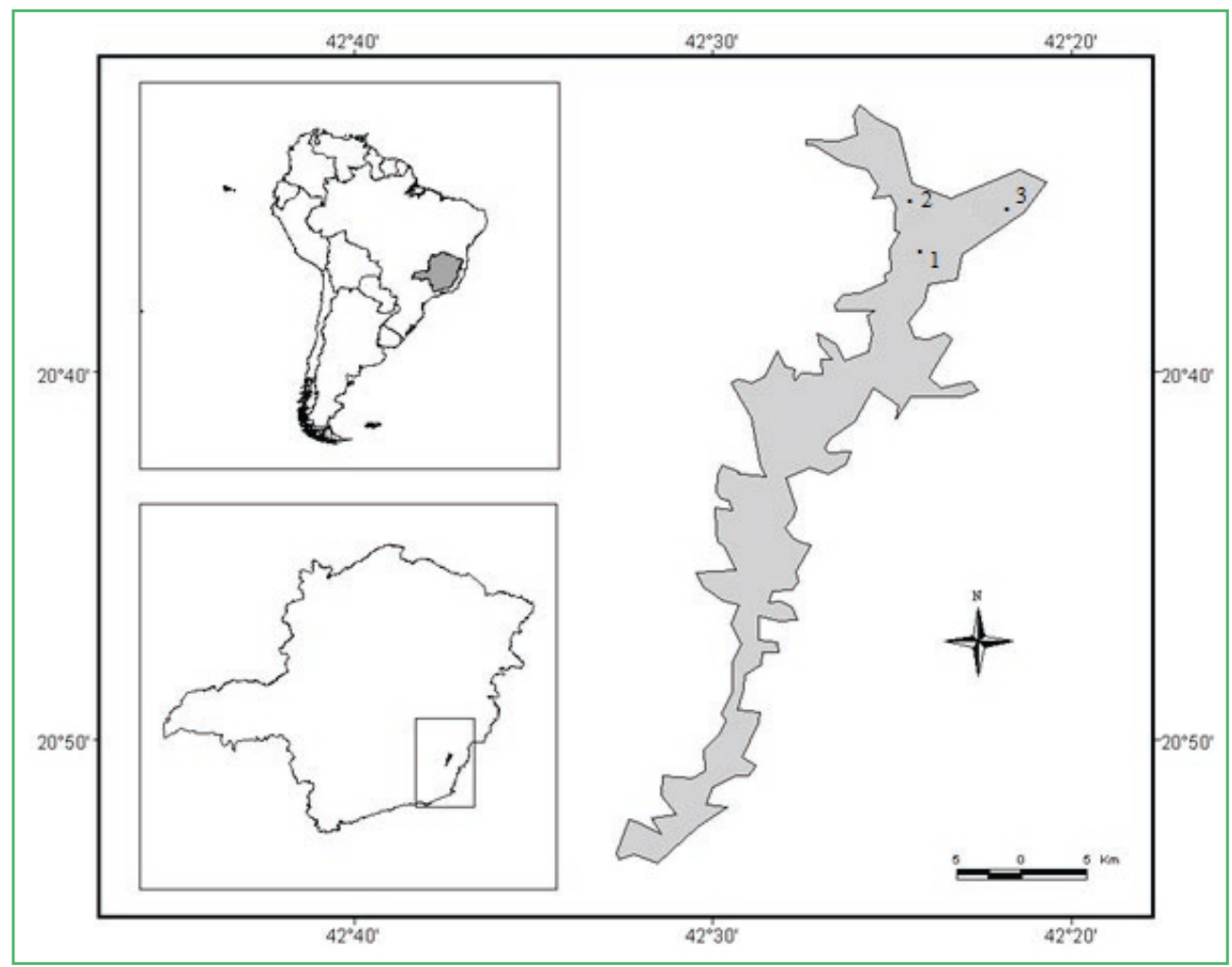

(CAIFA; SILVA, 2005). As áreas florestais apresentam graus variáveis de interferência antrópica, ocasionados pela retirada de parte da cobertura florestal, no final da década de 1950 e durante toda a década de 1960 (BENITES, 1998).

Em relação ao estágio sucessional, o norte do PESB apresenta um grande número de espécies secundárias iniciais tardias, colocando a floresta em um estágio mais inicial na sere sucessional (SILVA JÚNIOR, 2008), onde as localidades amostradas no presente estudo apresentam similaridade nesta característica, apesar da distância geográfica entre as mesmas (NUNES, 2009). Os sítios de amostragem do presente estudo foram selecionados considerando a existência prévia de trilhas do Projeto de Conservação e Manejo do Muriqui, por serem localidades do parque que nunca tiveram amostragem com foco em mamíferos terrestres de médio e grande porte, além de representarem a mesma fitofisionomia.

\section{Coleta de dados}

Foram realizados 12 meses de amostragem em três localidades (Vale Perdido, Matipó e Ararica) no norte do PESB (Figura 1). A amostragem da comunidade de mamíferos de médio e grande porte foi realizada por meio de oito armadilhas fotográficas analógicas (TrapaCâmera ${ }^{\circledR}$, modelo de filme $35 \mathrm{~mm}$ ). Assim, o total de unidades amostrais (armadilhas.dia) durante o período do estudo foi utilizado para representar o esforço efetivo de amostragem (curva do coletor).

As armadilhas fotográficas foram distribuídas em trilhas totalizando oito pontos em cada área de amostragem e permaneceram ativas, 24 horas por dia, durante 30 dias consecutivos. O mesmo protocolo foi utilizado nas três áreas. Os pontos de amostragem foram definidos em locais onde o posicionamento do equipamento e a qualidade dos registros não sofressem influências por declives, sendo instalados a uma distância mínima de $300 \mathrm{~m}$ entre pontos em cada área. As câmeras 
passaram por um sistema de rodízio entre áreas, sendo que a cada 30 dias uma nova área era amostrada.

\section{Análises de dados}

A hipótese de que existe diferença na riqueza de espécies entre os sítios de amostragem foi avaliada a partir de estimativas de riqueza geradas pelo procedimento Jackknife de $1^{\underline{a}}$ ordem (HELTSHE; FORRESTER, 1983). Para estimar a riqueza de espécies e obter o desvio padrão associado à estimativa para cada sítio de amostragem foi utilizado o programa Estimate $\mathrm{S}$ versão 6.0b1 (COLWELL, 2000). O intervalo de confiança (IC) foi calculado conforme Zar (1984) utilizando-se o desvio padrão associado à estimativa Jackknife 1 obtida pelo Estimate S. A riqueza estimada com o IC dos sítios de amostragem foram comparadas e a tomada de decisão foi feita por meio da inferência por intervalo de confiança ( $\mathrm{p}<0,05)$, procedimento similar à lógica dos testes de comparação de médias Teste t-student e ANOVA (ZAR, 1984).

Cada foto obtida em um intervalo de 24 horas de amostragem foi considerada como um registro independente. Se no mesmo ponto de amostragem, duas ou mais fotos de espécies de mamíferos de médio e grande porte que não apresentam marcas naturais (listras, manchas, pintas, etc.), foram registradas no mesmo intervalo de 24 horas, considerou-se apenas um registro.

Foi calculado o Índice de Abundância Relativa $(\mathrm{IAR})=\mathrm{S}_{\mathrm{i}} /(\mathrm{N} / 100)$, onde $\mathrm{S}_{\mathrm{i}}$ é o número de registros fotográficos independentes da espécie $i$, e $\mathrm{N}$ o esforço total e efetivo de amostragem em armadilhas-dia. $\mathrm{O}$ resultado foi tratado como uma medida de abundância relativa das espécies ao longo do período de amostragem. Em geral, assume-se que os índices indiretos sejam positivos e apresentam preferencialmente relação linear com a abundância real das populações (WILSON et al., 1996).
O número de registros independentes de mamíferos (variável independente) para cada área de amostragem é insuficiente para garantir o pressuposto de homogeneidade de variância e, consequentemente, o emprego de métodos estatísticos paramétricos. Por esse motivo optou-se pelo ajuste de modelos de distribuição da abundância, descritos por Magurran (2004), a partir dos resultados de abundância relativa das espécies para avaliar a estrutura da comunidade durante todo o período do estudo.

\section{Resultados}

\section{Riqueza e composição de espécies}

Foram obtidos 46 registros independentes de 10 espécies de mamíferos terrestres, sendo uma doméstica (Canis familiaris), pertencentes a três Ordens e sete Famílias (Tabela 1). A maior parte dos registros foi de táxons da Ordem Carnivora, sendo oito espécies e cinco Famílias. O total de registros do presente estudo representa $55 \%$ das espécies de mamíferos de médio e grande porte descritas para o PESB (LESSA et al., 2006; OLIVEIRA, 2007; PRADO, 2008).

Das espécies registradas, quatro integram alguma categoria de ameaça nas listas vermelhas ou categorias de interesse para a conservação. Leopardus pardalis, $L$. wiedii e Puma concolor figuram na categoria Vulnerável nas listas mineira (COPAM, 2010) e brasileira (MACHADO et al., 2008). O cateto (Pecari tajacu) também é classificado como Vulnerável em Minas Gerais (COPAM, 2010) e a única espécie que consta na lista vermelha global de espécies ameaçadas de extinção é L. wiedii, recentemente avaliada como espécie Quase Ameaçada na lista mundial (IUCN, 2011). 
TABELA 1: Lista das espécies de mamíferos, categoria de ameaça e número de registros independentes para os sítios de amostragem: VP (Vale perdido), MT (Matipó), AR (Ararica), localizadas no norte do Parque Estadual da Serra do Brigadeiro, Minas Gerais, Brasil.

\begin{tabular}{|c|c|c|c|c|c|c|}
\hline Táxon & Nome comum & $\begin{array}{c}\text { Categoria de } \\
\text { ameaça }\end{array}$ & $\mathbf{V P}$ & MT & $\mathbf{A R}$ & Total \\
\hline \multicolumn{7}{|l|}{ Ordem Rodentia } \\
\hline \multicolumn{7}{|l|}{ Família Cuniculidae } \\
\hline Cuniculus paca (Linnaeus, 1766) & Paca & $\mathrm{LC}$ & 2 & 1 & 3 & 6 \\
\hline \multicolumn{7}{|l|}{ Ordem Carnivora } \\
\hline \multicolumn{7}{|l|}{ Família Felidae } \\
\hline Leopardus pardalis (Linnaeus, 1758) & Jaguatirica & VU' ${ }^{\mathrm{BR}} ; \mathrm{VU} \mathrm{UG}^{\mathrm{MG}}$ & 1 & 2 & 2 & 5 \\
\hline Leopardus wiedii (Schinz, 1821) & gato-maracajá & $\mathrm{QA} ; \mathrm{VU}^{\mathrm{BR}} ; \mathrm{VU}^{\mathrm{MG}}$ & 1 & 0 & 1 & 2 \\
\hline Puma concolor Linnaeus, 1766 & onça-parda & VU ${ }^{\mathrm{BR}} ; \mathrm{VU}^{\mathrm{MG}}$ & 10 & 7 & 0 & 17 \\
\hline \multicolumn{7}{|l|}{ Família Canidae } \\
\hline Canis familiaris Linnaeus, 1758 & cachorro-doméstico & $\mathrm{LC}$ & 2 & 0 & 0 & 2 \\
\hline \multicolumn{7}{|l|}{ Família Mustelidae } \\
\hline Eira barbara Linnaeus, 1758 & Irara & $\mathrm{LC}$ & 0 & 2 & 0 & 2 \\
\hline \multicolumn{7}{|l|}{ Família Mephitidae } \\
\hline Conepatus semistriatus Boddaert, 1785 & Jaratataca & $\mathrm{LC}$ & 1 & 0 & 0 & 1 \\
\hline \multicolumn{7}{|l|}{ Família Procyonidae } \\
\hline Nasua nasua Linnaeus, 1766 & Quati & $\mathrm{LC}$ & 1 & 0 & 0 & 1 \\
\hline Procyon cancrivorus (G. [Baron] Cuvier, 1798) & mão-pelada & $\mathrm{LC}$ & 1 & 0 & 0 & 1 \\
\hline \multicolumn{7}{|l|}{ Ordem Artiodactyla } \\
\hline \multicolumn{7}{|l|}{ Família Tayassuidae } \\
\hline Pecari tajacu (Linnaeus, 1758) & Cateto & $\mathrm{VU}^{\mathrm{MG}}$ & 6 & 2 & 1 & 9 \\
\hline Total de registros & & & 25 & 14 & 7 & 46 \\
\hline
\end{tabular}

Legenda: $\mathrm{Vu}=$ vulnerável; $\mathrm{QA}$ = quase ameaçada; $\mathrm{LC}=$ não ameaçada. Categorias de ameaça de extinção, segundo a lista global da IUCN (2011), a lista nacional (BR) Machado et al. (2008) e mineira (MG) COPAM (2010).

O esforço efetivo de amostragem foi de 2.880 armadilhas.dia, sendo 960 unidades amostrais para cada um dos três sítios. Embora outras espécies de mamíferos terrestres com potencial registro por armadilhas fotográficas pudessem ter sido registradas, a análise da curva do coletor indica que a comunidade que utiliza as áreas amostradas foi bem representada (Figura 2). A comparação das estimativas para um mesmo esforço de coleta $(n=960)$ indica que os resultados para as três áreas parecem não terem sido influenciados pela amostragem (Figura 3).

As estimativas de riqueza de espécies para os sítios de amostragem foram: Vale Perdido 13,99 \pm 4,37 (média Jackknife $1 \pm$ intervalo de confiança), Ararica
$6,00 \pm 2,76$ e Matipó $6,00 \pm 1,96$. Estes resultados reforçam que a distribuição das espécies de mamíferos no PESB não é homogênea e que a intensidade de uso de algumas localidades é maior que outras. A comparação da composição de espécies entre os três sítios de amostragem indica apenas três registros comuns: L. pardalis, Cuniculus paca e P. tajacu. As espécies N. nasua, Procyon cancrivorus, Conepatus semistriatus e $C$. familiaris foram registrados apenas na área denominada Vale Perdido. Puma concolor teve registro na área Matipó e Vale Perdido, enquanto $L$. wiedii foi registrado nas áreas de amostragem Ararica e Vale Perdido. 
FIGURA 2: Riqueza observada e estimada de mamíferos terrestres de médio e grande porte no Parque Estadual da Serra do Brigadeiro, Minas Gerais, Brasil.

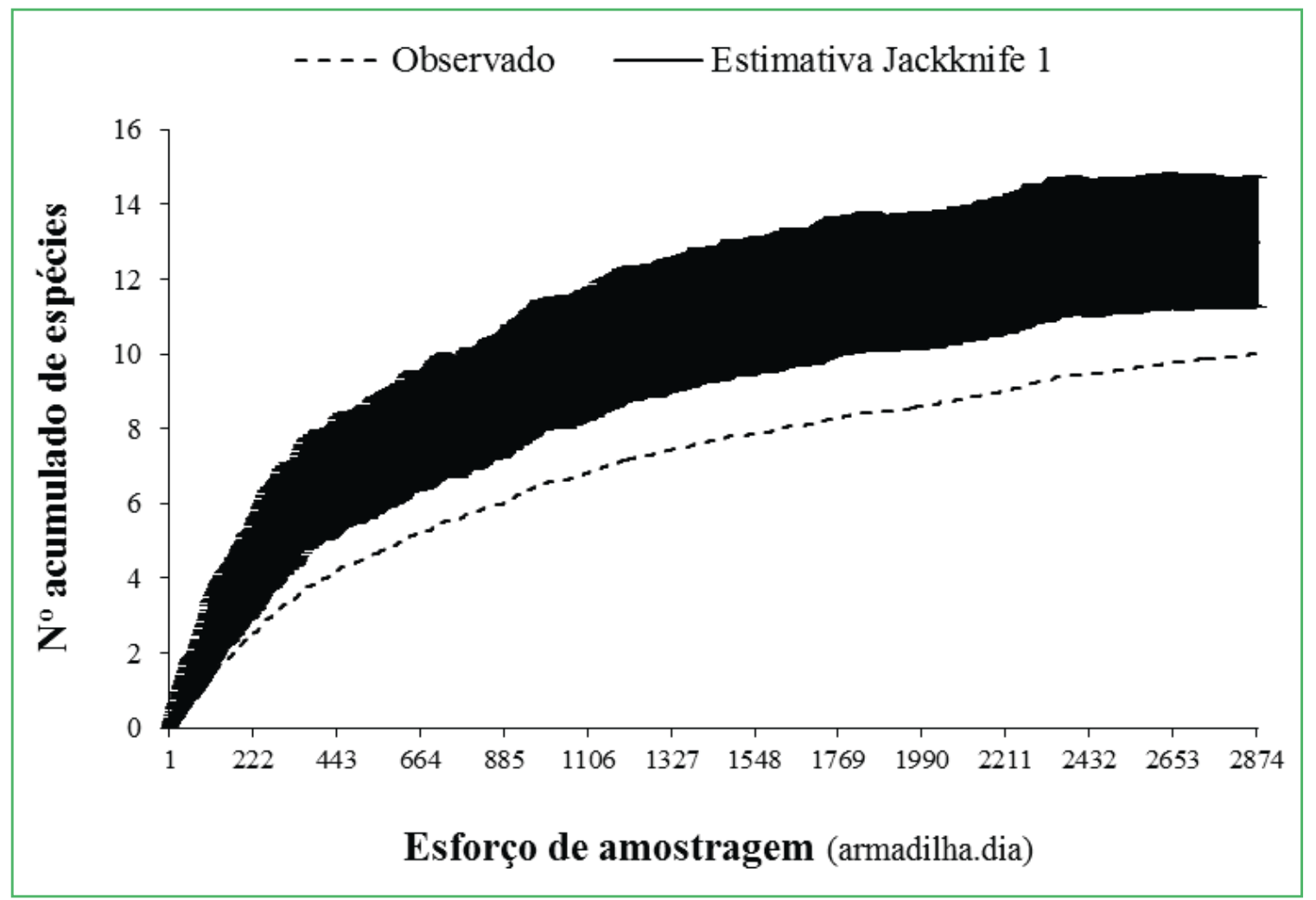

FIGURA 3: Estimativas de riqueza de espécies (Jackknife $1^{\text {a }}$ ordem) de mamíferos terrestres de médio e grande porte nos três sítios de amostragem.

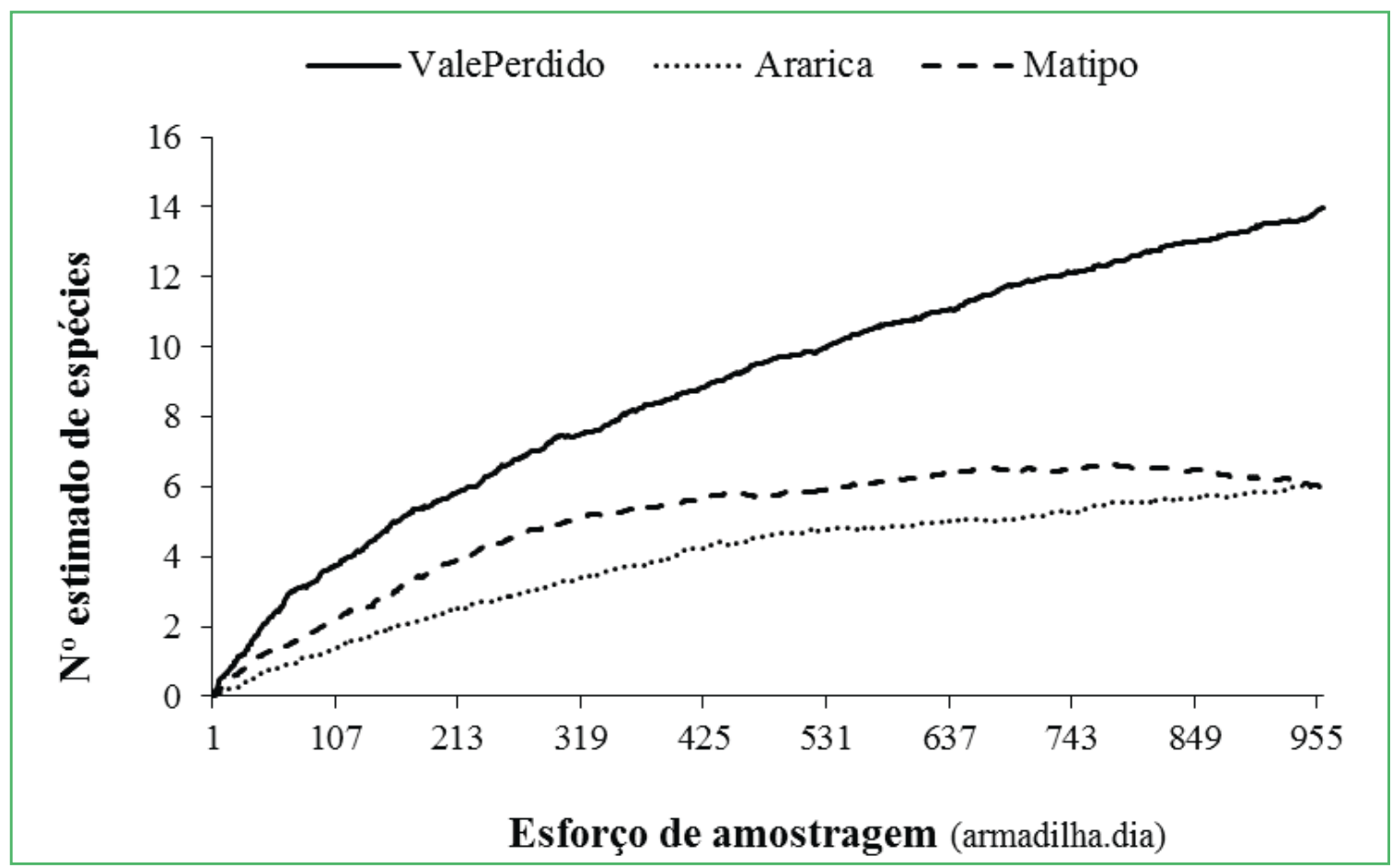




\section{Distribuição da abundância e estrutura da comunidade}

Com base no índice de abundância relativa calculado para cada espécie observa-se que a estrutura da comunidade de mamíferos de médio e grande porte do PESB é baseada numa série exponencial, com o dobro dos registros fotográficos de onça-parda (P. concolor) sobre as demais espécies da comunidade (Figura 4). Neste estudo foram fotografados pelo menos três indivíduos desta espécie em duas áreas de amostragem, identificados a partir da análise de marcas, cicatrizes e sexo, conforme descrito por Kelly et al. (2008).

\section{Discussão}

O conhecimento sobre a distribuição, riqueza e abundância das espécies de mamíferos terrestres de médio e grande porte do Parque Estadual da Serra do Brigadeiro, até então estava relacionada a estudos feitos na porção central do parque (LESSA et al., 2006; OLIVEIRA, 2007; PRADO, 2008). Os estudos supracitados relatam a ocorrência de 14 espécies para este grupo na região do parque. Das espécies registradas, cinco não tiveram registros no norte do PESB: Dasypus novemcinctus Linnaeus, 1758, Mazama americana Erxleben, 1777, Galictis cuja Molina, 1782, Cerdocyon thous Linnaeus, 1766 e Tamandua tetradactyla Linnaeus, 1758. Embora seja esperada a ocorrência também dessas espécies para esta região da Unidade de Conservação, devido a sua ampla distribuição geográfica no bioma da Mata Atlântica, provavelmente as mesmas apresentam baixa densidade local. Com o trabalho ora apresentado, apenas $C$. semistriatus não foi citado em nenhum dos estudos anteriores, sendo este o primeiro registro da espécie no interior do parque. Vale ressaltar que a riqueza de espécies observada para os estudos aqui comparados varia devido a diferenças ecológicas entre as áreas de amostragem, bem como às metodologias utilizadas e esforços amostrais (ver BEISIEGEL, 2009; SILVEIRA et al., 2010), o que também pode influenciar nos resultados de composição de espécies, como aqui observado.

A presença do cachorro doméstico na lista de espécies de mamíferos do PESB (OLIVEIRA, 2007; PRADO, 2008) e no presente estudo, chama atenção

FIGURA 4: Distribuição da abundância relativa das espécies de mamíferos terrestres de médio e grande porte registradas no Parque Estadual da Serra do Brigadeiro, Minas Gerais, Brasil. A linha pontilhada indica o ajuste do modelo exponencial $\left(\mathrm{r}^{2}=0,926\right)$.

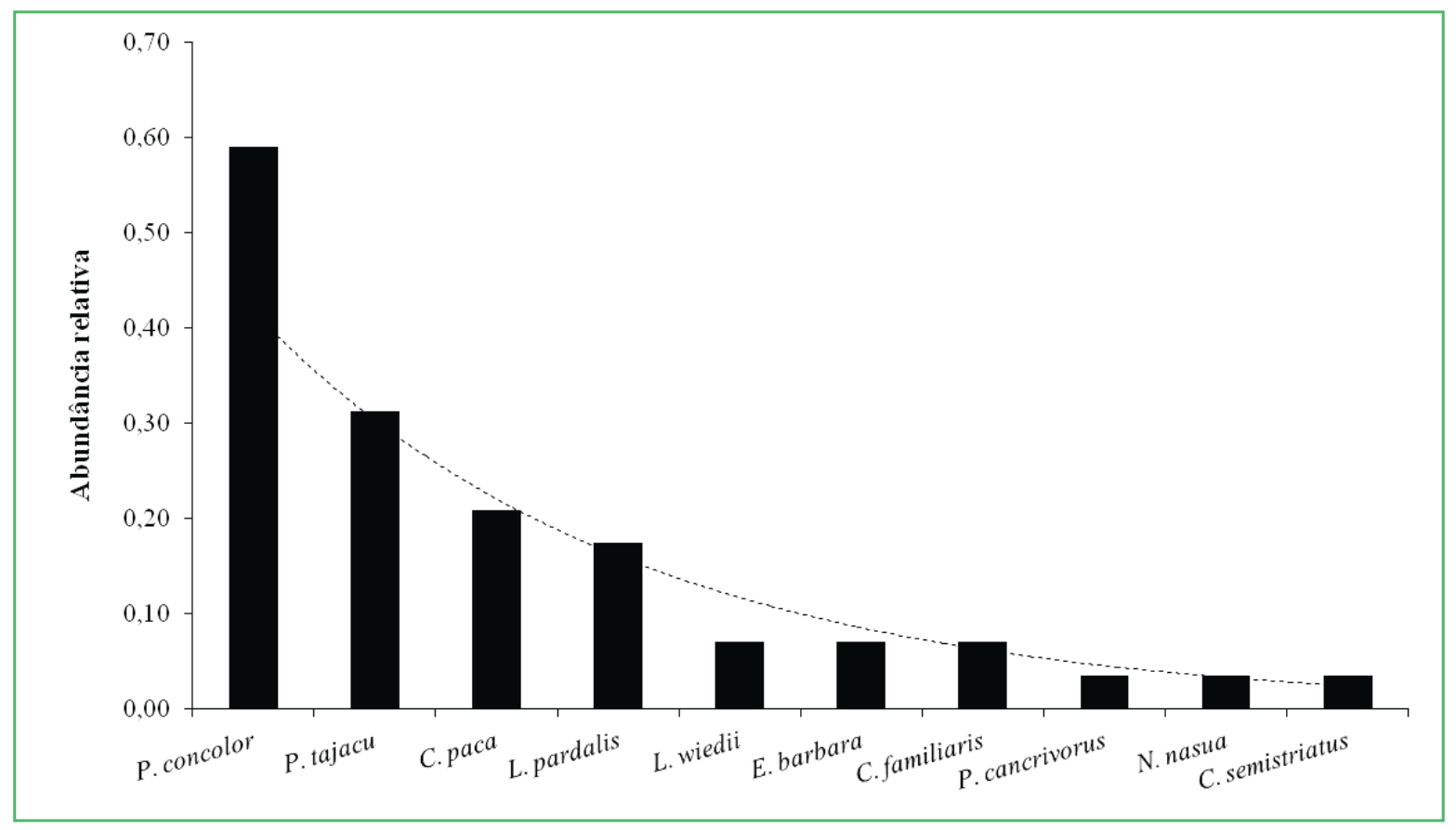


para possíveis impactos sobre a fauna silvestre. Cães domésticos são carnívoros oportunistas, competindo direta ou indiretamente na obtenção de alimentos com carnívoros silvestres (CAMPOS et al., 2007), além de serem importantes transmissores e vetores de doenças (COLEMAN et al., 1997). Canis familiaris tem sido registrado com certa frequência em áreas de Mata Atlântica (SRBEK-ARAUJO; CHIARELLO, 2008). No PESB, há relatos associados a um ataque a um indivíduo de Cebus nigritus Goldfuss, 1809 (OLIVEIRA et al., 2008). Galetti e Sazima (2006) num fragmento no estado de São Paulo observaram predação sobre animais de grande porte como veado-catigueiro (Mazama gouazoubira), paca (C. paca) e até mesmo primatas como o macaco-prego (C. nigritus) e bugio (Alouatta guariba Humboldt, 1812). O registro dessa espécie no interior da Unidade de Conservação indica que é possível que mudanças importantes ocorram na composição de espécies e abundância das populações locais, visto os impactos já descritos da mesma sobre espécies silvestres, principalmente associados à predação e à competição.

Observa-se que estudos que utilizam armadilhas fotográficas na região neotropical frequentemente apresentam um elevado número de espécies com poucos registros e algumas poucas espécies dominando em número de registros, o que influência o cálculo e interpretações sobre abundância relativa (SRBEKARAUJO; CHIARELLO, 2008; MAZZOLLI; HAMMER, 2008). No trabalho de Kasper et al. (2007), por exemplo, foram obtidos 1.605 registros de 23 espécies, sendo que apenas quatro (Dasyprocta azarae Lichtenstein, 1823, Sylvilagus brasiliensis Linnaeus, 1758, L. pardalis e P. tajacu) totalizaram $70 \%$ do total de registros fotográficos. Alves e Andriolo (2005) registraram 10 espécies em 250 registros fotográficos, dos quais, C. familiarias foi à espécie com maior abundância relativa, resultado similar ao apresentado por Srbek-Araujo e Chiarello (2008).

A ausência de algumas espécies de ampla distribuição geográfica, à presença do cachorro doméstico, e o histórico de modificações da paisagem local (e.g. caça, supressão vegetal e queimadas), pode ajudar a entender o pequeno número de espécies dominantes em contraposição a uma proporção maior de espécies com baixa densidade no PESB (LAURANCE; BIERREGARD, 1997; GALETTI; SAZIMA, 2006). Estes tipos de perturbações em ambientes naturais atuam na sobrevivência de espécies que apresentam uma baixa resiliência, e que são mais sensíveis a perturbações no habitat (BEGON et al., 1990), sendo apontados como as principais ameaças para a biodiversidade (DRUMMOND et al., 2005). O maior registro de espécies carnívoras no sistema, muito embora não se espere que uma espécie de grande porte e de topo de cadeia trófica esteja entre as dominantes de um sistema estável, pode ser um indicativo do estágio atual de conservação dos mamíferos de médio e grande porte do interior do PESB. Vale ressaltar que, mesmo com a distância relativamente pequena entre os pontos de amostragem, não seria esperado que o registro de $P$. concolor fosse mais frequente no presente estudo, visto que esta espécie apresenta uma área de vida maior se comparado aos demais mamíferos de médio e grande porte da comunidade apresentada (VIDOLIN; BRAGA, 2004). No entanto, é esperado que a distância entre pontos de amostragem aqui utilizado favorecesse o registro de espécies com menores áreas de vida (e.g. C. paca, Sylvilagus brasiliensis, Didelphis sp.) e que espécies que demandam áreas maiores seriam ocasionalmente registradas. $\mathrm{O}$ fato de se ter registrado três indivíduos de $P$. concolor em duas localidades distintas e o baixo número de registros de outras espécies reforça a existência de efeitos não controlados no presente estudo sobre os mamíferos de médio e grande porte com ocorrência para o PESB. Os resultados indicam que a comunidade de mamíferos provavelmente está em fase intermediária de estruturação após os distúrbios provocados pelas formas de uso e ocupação do território, supressão da vegetação, caça e incêndios florestais.

Alterações na abundância e na riqueza de mamíferos terrestres de médio e grande porte têm serias implicações para a conservação e manutenção de processos ecológicos como, por exemplo, dispersão e predação de sementes que afetam a dinâmica do sistema natural a médio e longo prazo (DIRZO; MIRANDA, 1990; REDFORD; EISENBERG, 1992; FRAGOSO, 1997; SILVA; TABARELLI, 2001). 
Apesar de alguns estudos terem observado a dispersão de sementes por espécies carnívoras (ROCHA et al., 2004), é possível que esta função ecológica no PESB esteja comprometida diante das cadeias tróficas da assembleia de mamíferos de médio e grande porte observada. A ausência de confirmação de registros históricos ou a baixa abundância de espécies de médio e grande porte (p. ex. Tapirus terrestris Linnaeus, 1758) e roedores (p.ex. Dasyprocta sp.) em fragmentos florestais e Unidades de Conservação pode ocasionar efeitos indiretos e de grande magnitude sobre a estrutura populacional de espécies vegetais que possuem frutos grandes como, por exemplo, palmeiras, sapotáceas e leguminosas (GALETTI et al., 2001; JORGE; PERES, 2005; JORDANO et al., 2006). Asquith et al. (1999) avaliaram que na ausência de cutias como dispersores de sementes de uma espécie de jatobá (Hymenaea courbaril L. Caesalpiniaceae) um número menor de plântulas foi recrutada. Estes resultados salientam a importância da presença desses animais para o incremento da probabilidade de sucesso no estabelecimento das sementes e plântulas em ambientes florestais, tornando estes ambientes mais ricos, complexos e dinâmicos (DIRZO; MIRANDA, 1990; REDFORD; EISENBERG, 1992; CUARÓN, 2000; GALETTI et al., 2003). O baixo número de registros de espécies de hábito alimentar preferencialmente frugívora e granívora no presente estudo, como $P$. tajacu e C. paca (PERES, 1999), reforça a hipótese acima, onde provavelmente as relações entre plantas e animas tenham sido afetadas diante da comunidade de granívoros/frugívoros observada. A comparação entre a distribuição dos registros nas três áreas amostradas indica a existência de diferenças em apenas uma área de amostragem em relação às demais. Maior número de registros fotográficos de espécies de mamíferos terrestres de médio e grande porte foi observado na área denominada como Vale Perdido em relação ao Matipó e Ararica. Possivelmente, existem características históricas, estruturais e espaciais que a torna, entre as três áreas, a mais importante para a conservação da mastofauna de médio e grande no PESB. Sua localização mais central na porção norte da Unidade de Conservação possivelmente reduz os efeitos de borda, que passam a atuar com menor intensidade, favorecendo o uso da mesma por espécimes de mamíferos de médio e grande porte.

Santos et al. (2004), em um estudo no Parque Nacional dos Aparados da Serra, atribuíram a ocorrência de um número maior de espécies em uma área central do parque à intensa fiscalização da região que inibe os efeitos negativos da caça, queimadas, desmatamento e pressões exercidas por espécies domésticas. Estas atividades estão intimamente associadas com a diminuição da riqueza de espécies (CHIARELLO, 2000; GALETTI et al., 2003; SANTOS et al., 2004; PAGLIA et al., 2006; MICHALSKI; PERES, 2007). Assim, é possível que esta área esteja atuando como áreanúcleo, onde os efeitos de borda são menos intensos. Esta hipótese sugere, portanto, menor probabilidade de extinção (ver PAGLIA et al., 2006) para as espécies que utilizam estás áreas em Unidades de Conservação. De qualquer forma, para avaliar esta hipótese é necessária a realização de novos estudos que busquem identificar o efeito de covariáveis sobre a riqueza, composição e distribuição espacial das espécies já que o PESB, por exemplo, apresenta gradientes altitudinais, forma longilínea e baixa conectividade com remanescentes florestais da região do entorno.

Por fim, conclui-se que o esforço amostral do presente estudo foi suficiente para avaliar a riqueza e a composição de espécies de mamíferos terrestres de médio e grande porte do PESB, muito embora, sabesse que quanto maior o esforço de coleta maior é a precisão do diagnóstico (HURLBERT, 1984; CALEY; SCHLUTER, 1997; KREBS, 1999; LANDE et al., 2000; DE MARCO JUNIOR; PAGLIA, 2003; MAGURRAN, 2004; FERRAZ et al., 2008; SILVEIRA et al., 2010). A provável extinção local de espécies importantes na dinâmica de ecossistemas florestais, que já haviam sido historicamente registradas no parque (comunicação pessoal de Renato Feio), e a presença de uma espécie doméstica de hábitos carnívoros indicam que o estado de conservação da comunidade de mamíferos analisada é crítico e demanda maior esforço de investigação para a recomendação de medidas específicas de manejo. Este estudo reforça as críticas apresentadas por Lima et al. (2005), sobre a necessidade de repensar os processos de criação e gestão de Unidades de Conservação já 
que o estado atual de boa parte das UCs de proteção integral não apresenta condições de efetiva proteção da biodiversidade. O PESB provavelmente já funciona como uma ilha de biodiversidade imersa em uma paisagem altamente fragmentada da região da Zona da Mata de Minas Gerais e, portanto, ações conservacionistas são prioritárias para buscar a manutenção de populações viáveis de mamíferos terrestres de médio e grande porte.

\section{Agradecimentos}

O presente estudo foi possível graças ao apoio logístico e financeiro do Museu de Zoologia João Moojen da Universidade Federal de Viçosa (MZUFV), do Programa Tropical Ecology, Assessment, and Monitoring (TEAM) Network, site Rio Doce, uma iniciativa da Conservação internacional com suporte financeiro da Gordon e Betty Moore Foundation, e da organização não governamental Ambiente Brasil. Ao Instituto Estadual de Florestas (IEF-MG) pela concessão da licença de pesquisa no interior da Unidade de Conservação. A Davi Feital, Leandro Moreira, Renato Totti, Anderson Ayres e a Letícia Bonfávaro pela ajuda durante as coletas de dados. A Maria Luiza e três revisores anônimos pelas sugestões e criticas ao manuscrito. Aos funcionários do PESB pelo apoio logístico.

\section{Referências}

ALVES, L. C. P.; ANDRIOLO, A. Camera trap used on the mastofaunal survey of Araras Biological Reserve, IEF-RJ. Revista Brasileira Zoociências, Juiz de Fora, v. 7, n. 2, p. 231-245, 2005.

ASQUITH, N. M.; TERBORGH, J.; ARNOLD, A. E.; RIVEROS, C. M. The fruits the agouti ate: Hymenaea courbaril seed fate when its disperser is absent. Journal of Tropical Ecology, Winchelsea, v. 15, n. 2, p. 229-235, 1999.

BEGON, M.; HARPER, J. L.; TOWNSEND, C. R. 1990. Ecology: individuals, populations and communities. 2. ed. Boston: Blackwell Scientific Publications, 1990. 1049 p.

BEISIEGEL, B. M. First camera-trap record of bush dogs in the state of São Paulo, Brazil. 2009. Disponível em $<$ http://www. canids.org/canidnews/12/Bush_dogs_in_Sao_Paulo.pdf $>$. Acesso em: 29 jun. 2010.

BENITES, V. M. Caracterização química e espectroscópica da matéria orgânica e suas relações com a gênese de solos da Serra do Brigadeiro, Zona da Mata Mineira. 1998. 123 f. Dissertação (Mestrado em Solos) - Universidade Federal de Viçosa, Viçosa. 1998.
CAIFA, A. N.; SILVA, A. F. Composição florística e espectro biológico de um campo de altitude do Parque Estadual da Serra do Brigadeiro, MG - Brasil. Rodriguésia, Rio de Janeiro, v. 56, n. 87, p. 163-173, 2005 .

CALEY, M. J.; SCHLUTER, D. The relatiomship between local and regional diversity. Ecology, New York, v. 78, n. 1, p. 70-80, 1997.

CAMPOS, C. B.; ESTEVES, C. F.; FERRAZ, K. M. P. M. B.; CRAWSHAW JÚNIOR, P. G.; VERDADE, L. M. Diet of free ranging cats and dogs in a suburban and rural environment, south eastern Brazil. Journal of Zoology, London, v. 273, p. 14-20, 2007.

CHIARELLO, A. G. Influência da caça ilegal sobre mamíferos e aves das matas de tabuleiro do norte do Estado do Espírito Santo. Boletim do Museu de Biologia Mello Leitão, Santa Teresa, v. $11 / 12$, p. 229-247, 2000.

COLEMAN, J. S.; TEMPle, S. A.; CRAVEN, S. R. Cats and wildlife: a conservation dilemma. 1997. Madison: University of Wisconsin. Disponível em $<$ http:www.wildlife.wisc.edu/extension/ carfly3.htm>. Acesso em: 18 jan. 2010.

COLWELL, R. K. 2000. Estimate S (version 6.0 b1.) - Statistical estimation of species richness and shared species from samples. University of Connecticut. Disponível em: $<$ http://viceroy.eeb. uconn.edu/estimates>.

COPAM - CONSELHO ESTADUAL DE POLÍTICA AMBIENTAL. 2010. Deliberação Normativa COPAM no 147, de 30 de abril de 2010: Aprova a Lista de Espécies Ameaçadas de Extinção da Fauna do Estado de Minas Gerais. Minas Gerais (Diário do Executivo), 04 maio 2010.

CUARÓN, A. D. A global perspective on habitat disturbance and tropical rainforest mammals. Conservation Biology, San Francisco, v. 14, n. 6, p. 1574-1579, 2000

DE MARCO JUNIOR, P.; PAGLIA, A. P. Estatística e interpretação de dados. In: CULLEN JUNIOR, L.; RUDRAN, R.; PADUA, C. V. (Org.). Métodos de estudos em biologia da conservação e manejo da vida silvestre. Curitiba: Editora UFPR, 2003. p. 211-235.

DIRZO, R; MIRANDA, A. Contemporary neotropical defaunation and forest structure, function and diversity sequel to John Terborgh. Conservation Biology, San Francisco, v. 4, n. 4, p. 444-447, 1990. DRUMMOND, G. M.; MARTIN, C. S.; MACHADO, A. B M.; SEBAIO, F. A.; ANTONINI, Y. Biodiversidade em Minas Gerais. Um atlas para sua conservação. Belo Horizonte: Fundação Biodiversitas, 2005. 222 p.

FERRAZ, G.; MARINELLI, C. E.; LOVEJOY, T. E.. Biological Monitoring in the Amazon: recent progress and future needs. Biotropica, Maiden, v. 40, n. 1, p. 7-10, 2008.

FIORELLO, C. V.; NOSS, A. J.; DEEN, S. L. Demography, hunting ecology, and pathogen exposure of domestic dogs in the Isoso of Bolivia. Conservation Biology, San Francisco, v. 20, n. 3, p. 762$771,2006$.

FRAGOSO, J. M. V. 1997. Tapir-generated seed shadows scaledependent patchiness in the amazon rain Forest. The Journal of Ecology, London, v. 85, n. 4, p. 519-529, 1997.

FROLICH, K.; CZUPALLA, O.; HAAS, L.; HENTSCHKE, J.; DEDEK, J.; FICKEL, J. Epizootiological investigation of canine distemper virus in free-ranging carnivores from Germany. Veterinary Microbiology, Geneva, v. 74, p. 283 -292, 2000. 
GALETTI, M.; COSTA, C.; GAZETTA, E. Effects of forest fragmentation antropogenic edges and fruit colors in the consumption of ornithochoric fruits. Biological Conservation, San Francisco, v. 111, p. 269-293, 2003.

GALETTI, M.; KEUROGHLIAN, A.; HANADA, L.; MORATO, M. I. Frugivory and seed dispersal by the lowland tapir (Tapirus terrestris) in Southeast Brazil. Biotropica, Maiden, v. 33, n. 4, p. 723-726, 2001.

GALETTI, M.; SAZIMA, I. Impacto de cães ferais e, um fragmento urbano de Floresta Atlântica no sudeste do Brasil. Natureza e Conservação, Curitiba, v. 4, p. 58-63, 2006.

HASKELL, J. P.; RITCHIE, M. E.; OLFF, H. Fractal geometry predicts varying body size scaling relationships for mammal and bird home ranges. Nature, London, v. 418, p. 527-530, 2002.

HELTSHE, J. F.; FORRESTER, N. E. Estimating species richness using the jackknife procedure. Biometrics, Washington, v. 39, p. 1-11, 1983 .

HENLE, K.; LINDENMAYER, D. B.; MARGULES, C. R.; SAUDERS, D. A., WISSEL, C. Species survival in fragmented landscapes: where are we now? Biodiversity and Conservation, New York, v. 13, p. 1-8, 2004.

HURLBERT, S. H. Pseudoreplication and the design of ecological field experiments. Ecological Monographs, Itchaca, v. 54, n. 2, p. 187-211, 1984.

IUCN. IUCN red list of threatened species. 2011. Gland and Cambridge: IUCN Species Survival Commission. Disponível em: $<$ http://www.redlist.org $>$. Acesso em: 20 set. 2011.

JORDANO, P.; GALETTI, M.; PIZO, M. A.; SILVA, W. Ligando frugivoria e dispersão à Biologia da Conservação. In: ROCHA, C F. D.; BERGALLO, H. G.; SLUYS, M.V.; ALVES, M. A. S. (Ed.). Biologia da Conservação: essências. São Carlos: Rima, 2006. p. 411-435.

JORGE, M. L. S. P.; PERES, C. A. Population density and home range size of red-rumped agoutis (Dasyprocta leporina) within and outside a natural Brazil nut stand in southeastern Amazonian. Biotropica, Maiden, v. 37, n. 2, p. 317-321, 2005.

KASPER, C. B.; MAZIM, F. D.; SOARES, J. B. G.; OLIVEIRA, T. G.; FABÍAN, M. M. Composição e abundância relativa dos mamíferos de médio e grande porte no Parque Estadual do Turvo, Rio Grande do Sul, Brasil. Revista Brasileira de Zoologia, Curitiba, v. 24, p. 4, p. 1087-1100, 2007.

KELLY, M. J.; NOSS, A. J.; DI BITETTI, M. S.; MAFFEI, L.; ARISPE, R. L.; PAVIOLO, A.; DEANGELO, C. D.; DI BLANCO, Y. E. Estimating puma densities from camera trapping across three study sites: Bolivia, Argentina, and Belize. Journal of Mammalogy, Lawrence, v. 89, n. 2, p. 408-418, 2008.

KIE, J. G.; BOWYER, R. T.; NICHOLSON, M. C.; BOROSKI, B. B.; LOFT, E. R. Landscape heterogeneity at differing scales: effects on spatial distribution of mule deer. Ecology, New York, v. 83, n. 2, p. 530-544, 2002.

KREBS, C. J. Ecological methodology. 2. ed. Menlo Park: Benjamin/Cummings, 1999. 620 p.

LANDE, R.; DEVRIES, P. J.; WALLA, T. When species accumulation curves intersect: implications for ranking diversity using small samples. Oikos, Copenhagen, v. 89, p. 601-605, 2000.
LAURANCE, W. F. Introduction and synthesis. Biological Conservation, Essex, v. 91, p. 101-107, 1999.

LAURANCE, W. F.; BIERREGARD, R. O. Tropical forest remmants: ecology, management and conservation of fragmented communities. Chicago: University of Chicago, 1997. 615 p.

LESSA, G.; MANDUCA, E. G.; PINTO, C. G. C.; MAGALHÃES, O. Caracterização da mastofauna do Parque Estadual da Serra do Brigadeiro, com vista à elaboração do Plano de Manejo. In: RELATÓRIO PARCIAL DO PLANO DE MANEJO DO PARQUE ESTADUAL DA SERRA DO BRIGADEIRO, 31, 2006, Belo Horizonte. Resumos... Belo Horizonte: IEF, 2006. Versão eletrônica. LIMA, G. S.; RIBEIRO, G. A.; GONCALVES, W. Avaliação da efetividade de manejo das unidades de conservação de proteção integral em Minas Gerais. Revista Árvore, Viçosa, v. 29, n. 4, p. 647-653, 2005.

MACHADO, A. B. M.; DRUMMOND, G. M.; PAGLIA, A. P. Livro vermelho da fauna brasileira ameaçada de extinção. In: MACHADO, A. B. M.; DRUMMOND, G. M.; PAGLIA, A. P. (Ed.). Vol. 2. Brasília: MMA; Belo Horizonte: Fundação Biodiversitas, 2008. 1420 p.

MAGURRAN, A. E. Measuring biological diversity. Oxford: Blackwell Publishing Company, 2004. 256 p.

MAZZOLLI, M.; HAMMER, M. L. A. Qualidade de ambiente para a onça-pintada, puma e jaguatirica na Baía de Guaratatuba, Estado do Paraná, utilizando os aplicativos Capture e Presence. Biotemas, Florianópolis, v. 21, n. 2, p. 105-117, 2008.

MICHALSKI, F.; PERES, C. A. Disturbance - mediated mammal persistence and abundance area relationships in Amazonia forest fragments. Conservation Biology, San Francisco, v. 21, n. 6, p. 1626-1640, 2007.

NOSS, R. F.; QUIGLEY, H. B.; HORNOCKER, M. G.; MERRIL, T.; PAQUET, P. C. Conservation biology and carnivore conservation in the Rocky Mountains. Conservation Biology, San Francisco, v. 10, p. 4, p. 949-963, 1996.

NUNES, A. V. 2009. Composição e conservação das comunidades de mamíferos terrestres de médio e grande porte do Parque Estadual da Serra do Brigadeiro. 2009. 50 f. Dissertação (Mestrado em Biologia Animal) - Universidade Federal de Viçosa, Viçosa. 2009.

OLIVEIRA, V. B. Uso de armadilhas de pegadas na amostragem da mastofauna em duas unidades de conservação nos biomas Cerrado e Mata Atlântica. 2007. 84 f. Dissertação (Mestrado em Zoologia) - Pontifícia Universidade Católica de Minas Gerais, Belo Horizonte. 2007.

OliveIRA, V. B.; LINARES, A. M.; CORRÊA, G. L. C.; CHIARELLO, A. G. 2008. Predation on the black capuchin monkey Cebus nigritus (Primates: Cebidae) by domestic dogs Canis lupus familiaris (Carnivora: Canidae), in the Parque Estadual da Serra do Brigadeiro, Minas Gerais, Brazil. Revista Brasileira de Zoologia, Curitiba, v. 25, p. 376-378, 2008.

PARDINI, R.; SOUZA, S. M.; BRAGA-NETO, R.; METZGER, J. P. The role of forest structure, fragment size and corridors in maintaining small mammal abundance and diversity in Atlantic Forest landscape. Biological Conservation, San Francisco, v. 124, p. 253-266, 2005.

PAGLIA, A. P.; FERNANDEZ, F. A. S.; DE MARCO, JR. P. Efeitos da fragmentação de habitats: quantas espécies, quantas populações, 
quantos indivíduos, e serão eles suficientes. In: ROCHA, C. F. D.; BERGALLO, H. G.; SLUYS, M.; ALVES, M. A. S. (Ed.). Biologia da Conservação: essências. São Carlos: Rima, 2006. p. 420-450.

PERES, C. A. The structure of nonvolant mammal communities in different Amazonian forest types. In: EISENBERG, J. F.; REDFORD, K. H. Mammals of the neotropicals. Chicago: Chicago University Press, 1999. p. 564-581.

PERES, C. A. Synergistic effects of subsistence hunting and habitat fragmentation on Amazonian forest vertebrates. Conservation Biology, San Francisco, v. 15, p. 6, p. 1490-1505, 2001.

PRADO, M. R. Impacto da BR - 482 na intensidade de uso do habitat e na diversidade de mamíferos no Parque Estadual da Serra do Brigadeiro, MG. 2008. 90 f. Dissertação (Mestrado em Ciências Florestais) - Universidade Federal de Viçosa, Viçosa. 2008.

REDFORD, K. H.; EISENBERG, J. F. Mammals of the neotropics: the southern cone. Chicago: Chicago University Press, 1992. 430 p.

RIBEIRO, C. A. N. 2003. Florística e fitossociologia de um trecho de floresta atlântica de altitude na fazenda Neblina, Parque Estadual da Serra do Brigadeiro, MG. 2003. 90 f. Dissertação (Mestrado em Botânica) - Universidade Federal de Viçosa, Viçosa. 2003.

ROCHA, V. J.; REIA, N. R.; SEKIAMA, M. L. Dieta e dispersão de sementes por Cerdocyon thous (Linnaeus) (Carnívora, Canidae), em um fragmento florestal no Paraná, Brasil. Revista Brasileira de Zoologia, Curitiba, v. 21, n. 4, p. 871-876, 2004.

SANTOS, M. F.; PELLAND, M.; TOMAZZONI, H. H.; HARTZ, S. M. Mamíferos carnívoros e sua relação com a diversidade de habitats no Parque Nacional dos Aparados da Serra, sul do Brasil. Iheringia, Série Zoologia, Porto Alegre, v. 94, n. 3, p. 235-245, 2004.
SILVA, M. G.; TABARELLI, M. Seed dispersal, plant recruitment and spatial distribuition of Bactris acanthocarpa Martius (Arecaceae) in a remnant of Atlantic Forest in northeast Brazil. Acta Oecologica, New York, v. 22, p. 259-268, 2001.

SILVA JUNIOR, W. M. 2008. Ecologia de florestas atlânticas com ocorrência do muriqui (Brachyteles spp.): diversidade, sucessão secundária e estrutura nutricional. 2008. 105 f. Tese (Doutorado em Botânica) - Universidade Federal de Viçosa, Viçosa. 2008.

SILVEIRA, L. F.; BEISIEGEL, B. M.; CURCIO, F. F.; VALDUJO, P. H.; DIXO, M.; VERDADE, V. K.; MATTOX, G. M. T.; CUNNINGHAM, P. T. M. Para que servem os inventários de fauna? Estudos Avançados, São Paulo, v. 24, n. 68, p. 173-207, 2010.

SRBEK-ARAujO, A. C.; CHIARELlO, A. G. Domestic dog in Atlantic Forest preserves of south-eastern Brazil: a camera trapping study on patterns of entrance and site occupancy rates. Brazilian Journal of Biology, São Carlos, v. 68, n. 4, p. 631-637, 2008.

VIDOLIN, G. P.; BRAGA, F. G. Ocorrência e uso de área por carnívoros silvestres no Parque Estadual do Cerrado, Jaguariaíva, Paraná. Caderno da Biodiversidade, Maringá, v. 4, n. 2, p. 29-36, 2004.

WEBB, C. O.; ACKERLY, D. D.; McPEEK, M. A.; DONOGHUE, M. J. Phylogenies and community ecology. Annual Review of Ecology and Systematics, Palo Alto, v. 33, p. 475-505, 2002.

WILSON, D. E.; COLE, F. R.; NICHOLS, J. D.; RUDRAN, R.; FOSTER, M. S. Measuring and monitoring biological diversity: standard methods for mammals. Washington: Smithsonian Press, 1996. 409 p

ZAR, J. H. Biostatistical Analysis. New Jersey: Prentice Hall International, 1984. p. 43-45. 